\title{
20 Jahre Ärztinnen und Ärzte für Umweltschutz
}

Peter Kälin ${ }^{a}$, Rita Moll ${ }^{b}$

a Dr. med., Präsident

b Dr. med. vet., Geschäftsleiterin
Seit ihrer Gründung vor 20 Jahren informieren die Ärztinnen und Ärzte für Umweltschutz (AefU) Ärzteschaft, Patientinnen und Patienten sowie die Öffentlichkeit über umweltbedingte Gefahren für die Gesundheit und mischen sich in die Umweltpolitik ein. Wir verstehen uns als Vertreterinnen und Vertreter des Fachbereiches Umweltmedizin und zugleich als unabhängige Nichtregierungsorganisation. Wir blicken zurück auf ein reichhaltiges Schaffen und werden nach der Jubiläumsfeier am 23. Juni 2007 in Basel $^{*}$ aufbrechen zu neuen Taten, im Gepäck die Erfahrung aus zwei Jahrzehnten und das Wissen um die krankmachenden Auswirkungen vieler Umweltbelastungen. Grosse Anstrengungen werden nötig sein, damit sich umweltbewusstes Handeln durchsetzt.

\section{Vorgeschichte}

Aus Sorge um das Waldsterben und die zunehmende Luftverschmutzung startete eine Handvoll Engagierte die Aktion «Luft ist Leben - Für Wald und Gesundheit - ein Aufruf von Ärzten an die eidgenössischen und kantonalen Behörden». Dieser Aufruf wurde in der Schweizerischen Ärztezeitung Mitte 1986 publiziert und an alle FMHMitglieder verschickt. Das Echo war gewaltig: 3533 Ärztinnen und Ärzte unterschrieben den Aufruf und taten damit ihre Betroffenheit kund. Der Aufruf mit konkreten Forderungen und die Unterzeichnerliste wurden dem Bundesrat und den Regierungen der Kantone durch Ärztedelegationen persönlich überbracht.

Es gab zu diesem Zeitpunkt im Raum Basel bereits einen sehr aktiven Verein mit dem Namen
Es gibt Themen, die sich wie ein roter Faden durch die 20 Jahre Vereinsgeschichte ziehen, andere sind erst vor kurzer Zeit hinzugekommen.

\section{Chemikalien}

So quasi als Mitgift hat der «alte» Basler Verein das Thema Chemikalien eingebracht, wie ein frühes Wartezimmerplakat und diverse Wartezimmerbroschüren belegen. Die AefU-Arbeitsgruppe «chemikalieninduzierte Krankheiten» hat sich sehr intensiv mit Umweltchemie beschäftigt und regelmässig ihre fundiert recherchierten Artikel in unserer Fachzeitschrift Oekoskop veröffentlicht. Unvergessen ist sicher noch die Aktion «Ich unterstütze den Aufruf < Verzicht aus Verantwortung> und verwende nach Möglichkeiten die Medikamente der FCKW-Hersteller nicht mehr». Eine, wie wir heute wissen, sehr erfolgreiche AefU-Kampagne.

Im letzten Jahr war «Chemie im Alltag» Thema unserer Fortbildungsveranstaltung «Forum Medizin und Umwelt», die wir seit vielen Jahren gemeinsam mit dem ISPM Basel für unsere Mitglieder organisieren und die sich grosser Beliebtheit erfreut.

Es war immer das Bestreben unseres Vereins, alle Mitglieder zu einer umweltgerechten Praxisführung zu motivieren. Früh schon wurden Empfehlungen zur Anwendung von Desinfektions- und Reinigungsmitteln in der Arztpraxis herausgegeben. Heute bieten die 7 Merkblätter «Ökologie in der Arztpraxis» eine praktische Hilfestellung.

\section{Gentechnologie}

Ein Thema, das uns all die Jahre stark in Anspruch genommen hat, ist die Gentechnologie. Wie andere Grosstechnologien birgt sie ein unbekannt grosses Gefahrenpotential. Auf wissenschaftlicher Ebene haben wir mit der Publikation «Gentechnologie und Nahrungsmittel» auf mögliche Folgen und Risiken aufmerksam gemacht und eine unabhängige Risikoforschung gefordert. Politisch haben Mitglieder unserer Arbeitsgruppe mit zahlreichen Auftritten für die Genschutz-Initiative geworben. Diese haben wir zwar nicht gewinnen können, aber ein paar Jahre \footnotetext{
«Ärzte für Umweltschutz» und 450 Mitgliedern. Es lag nahe und die Zeit war reif, sich zusammenzuschliessen: Die Bewegung «Luft ist Leben» und dieser bestehende Verein fusionierten und gründeten am 21. Juni 1987 in Bern den gesamtschweizerischen Verein «Ärzte für Umweltschutz». Die FMH hat dem jungen Sprössling zur Gründung einen Diavortrag zum Thema Waldsterben geschenkt, der rege benutzt wurde, um Öffentlichkeitsarbeit zu betreiben.

info@aefu.ch

www.aefu.ch

Korrespondenz:

Dr. med. vet. Rita Moll

52

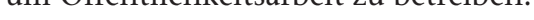

Offentlicher Festakt und Apéro am Samstag, 23. Juni 2007, von 18 bis 20 Uhr im Grossratssaal des Rathauses in Basel.
}

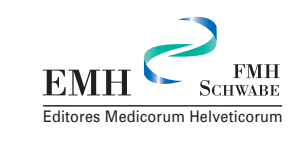


später ist es uns gemeinsam mit vielen anderen Organisationen mit der Gentechfrei-Initiative gelungen, die Schweizer Landwirtschaft vorläufig frei von Gentechnik zu halten.

Stark engagiert haben sich die AefU gegen die Patentierung von gentechnisch veränderten Lebewesen. Wir sind mit unseren Argumenten und Forderungen auf die Strasse gegangen - sowohl in München vor dem Europäischen Patentamt als auch in Bern vor dem Bundeshaus - und wir haben uns an Einsprachen (z.B. gegen das «Krebsmaus»-Patent) beteiligt.

\section{Atomenergie}

Neu aufgeflammt ist die Diskussion um neue Atomkraftwerke. Bereits 1990 haben die Ärztinnen und Ärzte für Umweltschutz klar Stellung bezogen. Gemeinsam mit unserer Schwesterorganisation PSR hiess schon damals die Botschaft: Strom ohne Atom. Ebenfalls gemeinsam mit der PSR haben wir 2003 ein Nationales Ärztekomitee gegründet und für die Volksinitiativen «Strom ohne Atom» und «Moratorium Plus» gekämpft. Aktuell bringt unser Wartezimmerplakat 2006 die Argumente auf den Punkt: Atomenergie bedroht Menschen und Umwelt.

Unsere Arbeitsgruppe Tschernobyl-Hilfe, die mittlerweile ein eigenständiger Verein geworden ist, hat mit ihrem Engagement Grosses vollbracht. Unzählige Male waren die Mitglieder der Arbeitsgruppe in Weissrussland. Sie haben unter anderem das Forschungsprojekt «Kindliche Schilddrüsenkarzinome» zusammen mit der Universität Bern durchgeführt. Sie haben ein Kinderhospiz auf die Beine gestellt und unterstützen es massiv, fachlich wie finanziell. Drei Konferenzen in Minsk zum Thema «Palliative care für Kinder» wurden bereits durchgeführt, eine weitere ist für nächstes Jahr geplant. Die Katastrophe im Reaktor von Tschernobyl und ihre Folgen sind nicht vergessen.

\section{Elektrosmog}

Eine weitere, sehr aktive Arbeitsgruppe der AefU befasst sich mit den gesundheitlichen Auswirkungen von elektromagnetischen Feldern. Zu Risiken und Nebenwirkungen können Sie die Mitglieder dieser Gruppe gerne befragen oder auf unserer Website den Internetratgeber «Elektrosmog im Alltag» konsultieren, der einfache und kostengünstige Tips zur Reduktion von Elektrosmog gibt.

Ungereimtheiten und Gefahren für die menschliche Gesundheit festzustellen heisst, politisch aktiv zu werden. Unsere Arbeitsgruppe hat aufgedeckt, wie die Mobilfunkindustrie versucht, die gesundheitlichen Risiken schönzu-

\section{0 ans d'existence des «Médecins en faveur de l'environnement» (MfE)}

Depuis 20 ans déjà - date de la fondation de leur association - les MfE sensibilisent le corps médical, les patients ainsi que la population aux risques de santé liés à l'environnement et s'engagent également en politique. Ils se considèrent comme les représentants du domaine spécifique de la médecine de l'environnement et, en même temps, comme une organisation non gouvernementale indépendante. Après avoir jeté un regard rétrospectif sur leur travail abondant et célébré le vingtième anniversaire de l'association ce 23 juin 2007, ils repartiront dans de nouveaux projets avec, pour bagage, une expérience de deux décennies et le savoir des effets néfastes de la pollution de l'environnement. II faudra encore de nombreux efforts pour aboutir à ce que les comportements écologiques l'emportent.

reden, Einfluss auf die Forschung nimmt und die Ärzteschaft zu beeinflussen versucht. Zentrale Forderungen der AefU sind eine industrieunabhängige Forschung, Senkung der Grenzwerte und ein Moratorium für den Weiterausbau der Mobilfunktechnologie.

\section{Lärm}

Die Broschüre «Lärm und Gesundheit» der AefU ist - wie in der Regel alle unsere Publikationen in drei Sprachen erschienen. Alle unsere Broschüren sind in enger Zusammenarbeit mit Fachpersonen der Schweizer Universitäten entstanden. Wir legen grossen Wert darauf, dass unsere Informationen für Kolleginnen und Kollegen, für die Behörden und für die Bevölkerung wissenschaftlich fundiert erarbeitet sind. Unser Faltblatt «Weniger Strassenlärm - mehr Gesundheit» gibt konkrete Anweisungen, wie Lärm vermindert werden kann, und leistet Hilfestellung für Betroffene.

Lärm bedeutet eine ernsthafte Gesundheitsgefährdung, die neuesten Erkenntnisse und unsere Forderungen sind im Oekoskop 1/07 publiziert.

Die Ärztinnen und Ärzte für Umweltschutz sind eine im Umweltschutzrecht zur Beschwerde berechtigte Organisation, und wir nehmen dieses Verbandsbeschwerderecht wohlüberlegt 


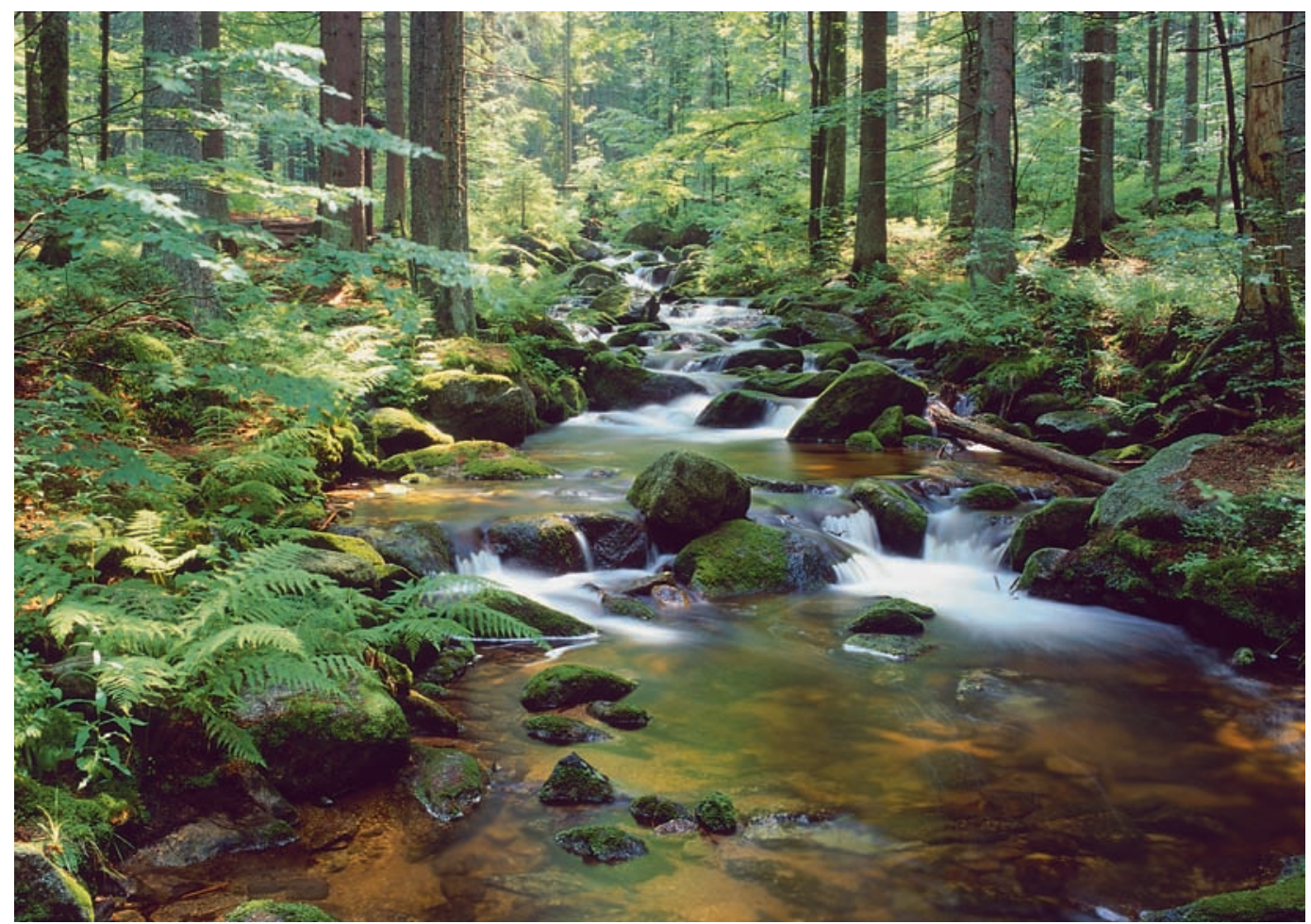

Eine intakte Natur wirkt sich positiv auf das Wohlbefinden der Menschen aus.

wahr - im Falle des Flughafens Zürich mit einer Beschwerde gegen das Betriebsreglement, in der Lärm ein Hauptthema ist. Wir verteidigen es auch gegen Angriffe, die an den Grundwerten eines Rechtsstaates rütteln.

\section{Luft/Verkehr}

Die Luftverschmutzung und damit verbunden der motorisierte Strassenverkehr haben die Aktivitäten der AefU in den letzten beiden Jahrzehnten stark geprägt. Unzählige Aktionen und Publikationen sind entstanden. Alles, was man über Luftverschmutzung und Gesundheit wissen muss, ist in einer AefU-Broschüre wissenschaftlich fundiert und gut verständlich zusammengefasst.

Den Verursachern des Feinstaubs haben wir den Kampf angesagt und hartnäckig mitgewirkt, dass in kürzester Zeit zumindest einmal Bewegung in die unhaltbare Situation der andauernden Grenzwertüberschreitungen gekommen ist. Luft ist Leben, dieses Motto gilt für uns noch immer, auch in Sachen Ozon. Alljährlich geben wir eine aktualisierte Sommersmog- und eine aktualisierte Feinstaubdokumentation heraus, die unsere Forderungen an die Behörden und die Politik für bessere Luft und Tips für die Bevölkerung enthält.

Es gibt wenige Volksinitiativen in der Schweiz, denen Erfolg beschieden ist. Für die Alpeninitiative haben wir das Beste gegeben.
Unser Verein und viele von uns ganz persönlich sind angetreten und haben ihren Beitrag geleistet für das Ja des Schweizer Volkes. Wenige Jahre später wurde die unsinnige Avanti-Forderung für eine weitere Gotthardröhre und die freie Fahrt für den motorisierten Strassenverkehr erfolgreich gebodigt.

\section{Klimawandel}

Unser Klima ist in Gefahr, das haben wir schon früh erkannt und bereits 1990 den Aufruf «Haut der Erde retten» zum Schutz der Erdatmosphäre gestartet. Wir dürfen schon für uns in Anspruch nehmen, Vordenkerinnen und Vordenker zu sein. Bei der Lektüre dieses Aufrufs wird klar, dass die damaligen Forderungen heute noch genauso aktuell sind: Drosselung des Verbrauchs an fossilen Brenn- und Treibstoffen, sparsame und rationelle Verwendung von Energie sowie Einsatz und Weiterentwicklung erneuerbarer Energiequellen und dass die Kernenergie keine Option im Kampf gegen die Klimaerwärmung darstellt, sind Stichworte daraus.

Wir stellen nicht nur Forderungen, wir bieten auch Lösungen an und führen Projekte durch. Im Auftrag von BAG und BAFU haben wir 3 Merkblätter zu den Auswirkungen der Klimaerwärmung verfasst und Empfehlungen zum Schutz bei Hitzewellen für Betagte und Pflegebedürftige erarbeitet. 
Die neu lancierte Klima-Initiative unterstützen wir tatkräftig - auch mit unserem brandneuen Wartezimmerplakat.

\section{Verbindung Gesundheitsförderung und Umweltschonung}

Aktuell gibt es neben den risikoorientierten Ansätzen, von denen bis jetzt die Rede war, auch ein weiteres Tätigkeitsfeld der AefU, nämlich die Verbindung oder Synergie von Gesundheitsförderung mit Umweltschonung. Mit dem Pilotprojekt «Leben in Bewegung» haben wir die Idee tägliche, regelmässige Bewegung, gekoppelt an umweltschonende Mobilität, aufgegriffen. Unsere Sektion beider Basel hat aktuell das Projekt «Gesund bewegt» mit ähnlicher Zielsetzung am Laufen - ein Siegerprojekt, ausgezeichnet mit dem Schweizer Mobilitätspreis prix pegasus.

Weitere spannende Aktivitäten verspricht unser neues, gemeinsam mit der Stiftung Landschaftsschutz Schweiz (SL) ins Leben gerufene Projekt «Paysage à votre santé». Landschaften mit bestimmten Qualitäten wirken sich positiv auf das Wohlbefinden der Menschen aus, diese Tatsache, die eine Literaturstudie des ISPM Bern im Auftrag von SL und AefU ans Licht gebracht hat, eröffnet neue Wege in der Gesundheitsförderung. Damit verbunden ist die hohe Gewichtung des Schutzes und der Aufwertung unserer Landschaften und der Umwelt.

\section{Und, und, und}

Wer jetzt glaubt, das seien so ungefähr die Aktivitäten der AefU in den letzten zwei Jahrzehnten gewesen, der täuscht sich, das ist längst nicht alles. Wir haben Einsitz in drei Eidgenössischen Kommissionen, wir haben Stellungnahmen geschrieben, Öffentlichkeitsarbeit geleistet,
Lobbyarbeit betrieben, Gespräche mit Behörden geführt, alles kann gar nicht aufgezählt werden.

Wir sind vernetzt mit anderen Umweltorganisationen, Hochschulen und Ämtern und nutzen die sich bietenden Synergien für eine zielgerichtete Sachpolitik. Wir sind Teil der FMH, und als Mitglied der «International Society of Doctors for the Environment» (ISDE) bewegen wir uns auch auf dem internationalen Parkett.

Im neuesten Projekt «Umweltmedizinisches Beratungsnetz» wird mit praktizierenden Kolleginnen und Kollegen und wissenschaftlicher Begleitung eine unabhängige medizinische Abklärungs- und Beratungsstelle für Betroffene aufgebaut.

\section{Ausblick}

In all den 20 Jahren haben engagierte Ärztinnen und Ärzte im Zentralvorstand, in den Sektionen, in Arbeitsgruppen, als Einzelpersonen - unterstützt von Geschäftsleitung und Sekretariat Gefahren für die Gesundheit, die aus Umweltbelastungen resultieren, öffentlich bekanntgemacht. Es wurde vieles bewegt, und es braucht die AefU weiterhin als Gegengewicht zu galoppierender Rücksichtslosigkeit und masslosem Gewinnstreben unserer Zeit. Nach unserer Jubiläumsfeier werden wir aufbrechen $\mathrm{zu}$ neuen Taten. In unserem Gepäck die Erfahrung von zwei Jahrzehnten und das Wissen um die krankmachenden Auswirkungen vieler Umweltbelastungen. Beides wollen wir vermitteln und Verbesserungen zum Schutze der Gesundheit einleiten. Wir sind uns bewusst, dass es grosse Anstrengungen aller braucht, damit sich umweltbewusstes Handeln durchsetzt. Neue Mitglieder sind in unserem Verein herzlich willkommen. 